\title{
ADAM10 attenuates the development of abdominal aortic aneurysms in a mouse model
}

\author{
RENFENG QIU ${ }^{1,2}$, SHUXIAO CHEN ${ }^{1}$, PEIXIAN GAO ${ }^{1,3}$, KUN LUO $^{1}$, \\ XUEDONG FENG ${ }^{3}$, HAI YUAN ${ }^{1,3}$, XUEJUN WU ${ }^{1,3}$ and GANG LI ${ }^{1,3}$ \\ ${ }^{1}$ Department of Vascular Surgery, Shandong Provincial Hospital, Cheeloo College of Medicine, Shandong University, \\ Jinan, Shandong 250021; ${ }^{2}$ Department of Vascular Surgery, Shouguang People's Hospital of Shandong, \\ Jinan, Shandong 262700; ${ }^{3}$ Department of Vascular Surgery, Shandong Provincial Hospital Affiliated to \\ Shandong First Medical University, Jinan, Shandong 250021, P.R. China
}

Received February 5, 2021; Accepted July 23, 2021

DOI: $10.3892 / \mathrm{mmr} .2021 .12414$

\begin{abstract}
An abdominal aortic aneurysm (AAA) is a life-threatening disease associated with a high mortality rate. At present, surgery or minimally invasive interventions are used in clinical treatment, especially for small aneurysms. However, the benefits of surgical repair are not obvious, and AAA ruptures can be prevented by aneurysm therapy to inhibit the growth of small aneurysms. Therefore, evaluating effective drugs to treat small AAAs is urgently required. Chronic inflammation is the main pathological feature of aneurysmal tissues. The aim of the present study was to investigate the protective role and underlying mechanism of ADAM metallopeptidase domain 10 (ADAM10). In the present study, a mouse model of AAA was established via porcine pancreatic elastase perfusion for 5 min per day for 14 days. ADAM10 $(6 \mathrm{mg} / \mathrm{kg})$ was injected intraperitoneally following 3 days of porcine pancreatic elastase perfusion in the ADAM10 group and the treatment continued for 10 days. The maximum inner luminal diameters of the infrarenal abdominal aortas were measured using an animal ultrasound system. The levels of high mobility group box 1 (HMGB1) and soluble receptor for advanced glycosylation end products in serum samples were measured by ELISA. Hematoxylin and eosin and elastin van Gieson staining were performed to observe morphology, integrity of the elastin layers and elastin degradation. CD68 expression was detected by immunohistochemical staining. Reverse transcription-quantitative PCR and western blotting were used
\end{abstract}

Correspondence to: Professor Gang Li or Professor Xuejun Wu, Department of Vascular Surgery, Shandong Provincial Hospital, Cheeloo College of Medicine, Shandong University, 324 Jingwuweiqi Road, Jinan, Shandong 250021, P.R. China

E-mail: ligangdoctor627@163.com

E-mail: sdwuxuejun@163.com

Key words: ADAM metalloprotease domain 10, high mobility group box 1, receptor for advanced glycosylation end products, $\mathrm{NF}-\kappa \mathrm{B}$, abdominal aortic aneurysm for detection of mRNA and protein levels. The gelatinolytic activities of MMP-2 and MMP-9 were quantified via gelatin zymography analysis. These results showed that ADAM10 inhibited $\mathrm{HMGB} 1 / \mathrm{RAGE} / \mathrm{NF}-\kappa \mathrm{B}$ signaling and MMP activity in the pathogenesis of pancreatic elastase-induced AAA, which provide insight into the molecular mechanism of AAA and suggested that ADAM10 may be a potential therapeutic target for AAA.

\section{Introduction}

An abdominal aortic aneurysm (AAA) is diagnosed if the diameter of an artery is $>30 \mathrm{~mm}$ or if it has increased by $>50 \%$ compared with the adjacent normal aorta (1). The mortality rate of patients with an AAA rupture is 65-85\%; currently, surgery or minimally invasive interventions are used for clinical treatment (2). However, randomized trials have reported that, although selective repair of AAAs can prevent rupture of the aneurysms and related deaths, it causes more perioperative deaths than any other common types of surgery or vascular surgery (3). Especially for small aneurysms, the benefits of surgical repair are not obvious, and an AAA rupture is likely to be prevented by aneurysm therapy to inhibit the growth of small aneurysms (4). Therefore, examining effective drugs to treat small AAAs is urgently required.

Chronic inflammation is the main pathological feature in aneurysmal tissues, involving the infiltration of inflammatory cells, elastic fiber degradation of the extracellular matrix (ECM), neovascularization and apoptosis, dysfunction of vascular smooth muscle cells and the release of a range of proteolytic enzymes, such as MMPs, oxidation-derived free radicals, cytokines and related products (5). Macrophages are the dominant factor in the production of MMPs during the inflammation of the media and adventitia. These MMPs cause the degradation of the ECM (6).

Receptor for advanced glycosylation end products (RAGE) is a cell-surface multiligand receptor of the immunoglobulin superfamily (7). As an important regulator of chronic and sustained inflammation, RAGE serves a key role in the development and progression of pathological states, such as cardiovascular disease, diabetic vascular complications, 
Alzheimer's disease (AD), cancer and a range of inflammatory diseases $(8,9)$. RAGE exists as a full-length isoform (fl-RAGE) and a soluble isoform (sRAGE). fl-RAGE consists of extracellular domains, a short transmembrane helix and a cytosolic C-terminal short tail. The cytosolic short tail domain is critical, and is responsible for transducing downstream signaling from the extracellular space to the intracellular space (10). sRAGE is one of the truncated forms of RAGE and is found in the serum in humans. As an endogenous competitor of RAGE, increased levels of sRAGE serve a critical role within the modulatory network of the ligand/RAGE axis and acts to alleviate the inflammatory response. As a biomarker of RAGE-mediated pathogenesis, sRAGE can reflect RAGE activity in the opposite direction (11). Furthermore, ectodomain shedding by proteolysis of RAGE is a means of sRAGE production.

High mobility group box 1 (HMGB1), one of the ligands of RAGE and a proinflammatory factor, is an evolutionarily highly conserved nuclear, nonhistone DNA-binding protein that exerts a proinflammatory role in a series of pathologies by regulating the release of inflammatory cytokines, activating macrophages and increasing vascular permeability (12). HMGB1 is involved in the pathogenesis of inflammatory conditions via its interactions with pivotal transmembrane receptors, including RAGE and Toll-like receptor-4 $(13,14)$. Extracellularhyperexpression of HMGB1 has been documented in various diseases, such as cardiovascular disease, septic shock, rheumatoid arthritis, atherosclerosis, traumatic brain injury and ischemia/reperfusion injury (15-17). After binding to membrane receptors, HMGB1 activates downstream NF- $\mathrm{NB}$ and further stimulates the expression of related inflammatory factors, such as oxidative stress and proinflammatory cytokines, which has been observed in AAAs (18).

ADAM metallopeptidase domain 10 (ADAM10) is one of the most closely studied members of the ADAM family, which has been shown to participate in the inflammatory response, and it is a target for the treatment of inflammatory diseases (19). As a membrane-anchored protease, ADAM10-mediated shedding is essential for numerous biological processes, such as cell migration, proliferation and $(20,21)$. ADAM10 is also responsible for RAGE cleavage, and binding to its ligand HMGB1 promotes RAGE shedding (22). Moreover, targeting of ADAM10 is a therapeutic strategy for the treatment of AD and prion disease (23), but its role in AAAs remains unknown.

Therefore, in the present study, we hypothesized that ADAM10 may attenuate the inflammatory progression of AAAs by inhibiting HMGB1/RAGE/NF- $\kappa$ B signaling. The current study examined the effect of ADAM10 on the expression levels of HMGB1 and RAGE, and the activation of NF- $\mathrm{NB}$ in an experimental model of AAAs in C57BL/6 mice.

\section{Materials and methods}

Animal groups. A total of 21 male C57BL/6 wild-type mice (age, 8-12 weeks; weight, 20-26 g) were purchased from the Experimental Animal Center of Shandong University (Jinan, China). The mice were randomly divided into the following groups ( $n=7$ per group): i) Sham-operated (Sham) group; ii) AAA model (AAA) group; and iii) ADAM10-treated (ADAM10) group. Mice were housed at $20-25^{\circ} \mathrm{C}$ and a humidity of $40-70 \%$ with a 12:12 h light-dark cycle. Water and food were given ad libitum and were provided by the Experimental Center of Shandong Provincial Hospital Affiliated with Shandong University. This study was performed according to the Care and Use of Laboratory Animals guidelines (24). The experimental protocols were approved by the Ethical Review Board of Shandong University (approval no. 2018-015). The animal experiments were performed between 01/2019 and $12 / 2019$.

Establishment of the AAA model and administration of ADAM10. The AAA model was established as described previously (25). Briefly, mice were anesthetized via $2.5 \%$ isoflurane inhalation. A para-abdominal median incision was made to expose the lower abdominal aorta to the bifurcation of the abdominal aorta, and a $10-\mathrm{mm}$ segment of the infrarenal abdominal aorta was exposed. Subsequently, $30 \mu \mathrm{l}$ porcine pancreatic elastase $(1.5 \mathrm{U} / \mathrm{ml}$; cat. no. E1250; Sigma-Aldrich; Merck KGaA) was perfused intraluminally for 5 min via a PPE-10 catheter that had been inserted into the aorta. PBS was used as a control in the Sham group. ADAM10 (6 mg/kg; cat. no. 936-AD; R\&D Systems, Inc.) was injected intraperitoneally after 3 days of porcine pancreatic elastase perfusion in the ADAM10 group and the treatment continued for 10 days. At the end of the study, the mice were euthanized under $\mathrm{CO}_{2}$ exposure (flow rate of $\mathrm{CO}_{2}, 2 \mathrm{1} / \mathrm{min}$; air displacement rate, $20 \% / \mathrm{min}$ ). Blood was collected after mouse euthanasia via cardiac puncture. Blood samples were separated via centrifugation at $3,000 \mathrm{x} \mathrm{g}$ at $4^{\circ} \mathrm{C}$ for $10 \mathrm{~min}$ and stored at $-80^{\circ} \mathrm{C}$. The aortas were embedded in Tissue-Tek O.C.T. compound at $-80^{\circ} \mathrm{C}$ or stored at $-80^{\circ} \mathrm{C}$ for protein and reverse transcription-quantitative (RT-q) PCR detection.

Measurements of the abdominal aortic diameter. The maximum inner luminal diameters of the infrarenal abdominal aortas were measured using an animal ultrasound system (FUJIFILM VisualSonics, Inc.). Measurements and analysis of dilation were carried out on days 3, 7 and 14 after AAA induction by two experienced operators who did not know their group assignment. AAA was defined as dilatation $>50 \%$ of the average diameter of the aorta.

ELISA examination. The levels of HMGB1 and sRAGE in the serum samples were measured using ELISA kits (HMGB1, cat. no. CSB-E08225, Cusabio Technology LLC; sRAGE, cat. no. MRG00, R\&D Systems, Inc.) according to the manufacturer's instructions. Sample diluent (100 $\mu \mathrm{l})$ and serum $(100 \mu \mathrm{l})$ were added to the wells and incubated at $37^{\circ} \mathrm{C}$ for $2 \mathrm{~h}$. Subsequently, biotin-labeled antibody working fluid $(100 \mu \mathrm{l}$; $1 \mathrm{X}$ antibody) was added to each well and incubated at $37^{\circ} \mathrm{C}$ for $1 \mathrm{~h}$. Then, the plate was washed three times with 0.01 M PBS. A total of $100 \mu \mathrm{l}$ substrate solution was added and incubated at room temperature for $1 \mathrm{~h}$. TMB color developing agent $(90 \mu \mathrm{l})$ was added to each well and incubated at $37^{\circ} \mathrm{C}$ for $30 \mathrm{~min}$ in the dark, and then $50 \mu \mathrm{l}$ termination solution was added. The optical density value was measured using a ThermoMultiskan GO microplate reader (1510-01981; Thermo Fisher Scientific, Inc.) at $450 \mathrm{~nm}$.

$H \& E$ and Elastin van Gieson (EVG) staining. The aortic specimens were fixed in $4 \%$ paraformaldehyde for $24 \mathrm{~h}$ at 
room temperature, then cut into 5- $\mu \mathrm{m}$ serial sections using a freezing microtome. $\mathrm{H} \& \mathrm{E}$ and EVG staining were performed following a standard protocol. The sections were stained with hematoxylin for $3 \mathrm{~min}$ and eosin solution for $15 \mathrm{sec}$ at room temperature. The sections were stained with Weigerts Resorcin Fuchsin solution for 45 min and Van Gieson solutions for $5 \mathrm{~min}$ at room temperature for EVG staining. The integrity of the elastin layers and elastin degradation were observed by light microscopy (BX3-CBH; OLYMPUS cellSens Standard 1.16; Olympus Corporation; magnification, x50 and x200).

Immunohistochemical staining. The aortic specimens were fixed in $4 \%$ paraformaldehyde for $24 \mathrm{~h}$ at room temperature, and then after washing and dehydration they were embedded by optimal cutting temperature compound and stored in $-80^{\circ} \mathrm{C}$. The sections were cut into $5-\mu \mathrm{m}$ serial sections by a freezing microtome as previously described (26). The sections were incubated with primary polyclonal antibodies against CD68 (1:400; cat. no. ab125212; Abcam) at $4^{\circ} \mathrm{C}$ overnight and blocked with goat serum (1:10; cat. no. ZLI-9021; ZSGB-BIO) for $30 \mathrm{~min}$ at room temperature. The sections were incubated with secondary HRP-goat anti-rabbit $\mathrm{IgG}$ antibodies for $30 \mathrm{~min}$ at room temperature by the rabbit polymer detection system (1X; cat. no. PV-6001; ZSGB-BIO). The sections were then stained with DAB reagent $(1 \mathrm{X})$ for $3 \mathrm{~min}$ at room temperature. Stained sections were observed by light microscopy (BX3-CBH; OLYMPUS cellSens Standard 1.16; Olympus Corporation).

RT-qPCR analysis. Total RNA was extracted from the abdominal aorta tissue using TRIzol ${ }^{\circledR}$ reagent (Invitrogen; Thermo Fisher Scientific, Inc.) according to the manufacturer's protocol. cDNA was synthesized from $1 \mu \mathrm{g}$ total RNA according to the manufacturer's protocol by PrimeScript RT reagent kit gDNAEraser (cat. no. RR047A; Takara Bio, Inc.) in a $20 \mu \mathrm{l}$ reaction volume. RT-qPCR was performed with $2 \mu \mathrm{l}$ cDNA and gene-specific primers in a final $20 \mu \mathrm{l}$ reaction system. The forward and reverse primers designed by Takara Bio, Inc. are shown in Table I. Amplification was performed using a SYBR Premix Ex Taq kit (Takara Bio, Inc.), and the thermocycling conditions were as follows: Initial denaturation at $95^{\circ} \mathrm{C}$ for $30 \mathrm{sec}$, followed by 45 cycles of $95^{\circ} \mathrm{C}$ for $3 \mathrm{sec}$ and $60^{\circ} \mathrm{C}$ for $30 \mathrm{sec}$. The relative mRNA expression was assessed using the $2^{-\Delta \Delta \mathrm{Cq}}$ method (27). The relative expression of the target gene was normalized to that of $\beta$-actin.

Western blot analysis. Mouse aortas were harvested for protein extraction by RIPA buffer (cat. no. P0013B; Beyotime Institute of Biotechnology) and a BCA protein concentration kit (cat. no. P0010; Beyotime Institute of Biotechnology) was used for protein determination. A total of $30 \mu \mathrm{g}$ protein extract was loaded per lane and separated by $10 \%$ SDS-PAGE and then electrotransferred to PVDF membranes (MilliporeSigma), which were blocked with 5\% skimmed milk in PBS-0.05\% Tween-20 (PBST) at room temperature for $1 \mathrm{~h}$. Next, primary antibodies against HMGB1 (1:1,000; cat. no. ab18256; Abcam), RAGE (1:1,000; cat. no. ab3611; Abcam), phosphorylated (p)-NF-кB p65 (Ser536; 93H1; 1:1,000; cat. no. 3033; Cell Signaling Technology, Inc.), NF-кB p65 (1:1,000; cat. no. ab16502; Abcam) and GAPDH (1:2,000; cat. no. ab181602; Abcam) were applied at $4^{\circ} \mathrm{C}$ overnight.
The membranes were washed three times, for $10 \mathrm{~min}$ each time, in PBST (0.1\% Tween-20). The appropriate horseradish peroxidase-conjugated secondary antibodies were applied at room temperature for $2 \mathrm{~h}$ including Goat Anti-Rabbit $(1: 3,000$; cat. no. ZB-5305; ZSGB-BIO) and Rabbit Anti-Mouse IgG H\&L (1:2,000; cat. no. ZB-2301; ZSGB-BIO). The bands were visualized using ECL assays (Thermo Fisher Scientific, Inc.) and assessed via semi-quantification of the optical density with Multi Gauge v3.2 software (FUJIFILM Wako Pure Chemical Corporation). GAPDH was used as an internal reference.

Gelatin zymography analysis. Tissue extracts were isolated according to the procedures described for the western blot analysis. Proteins were extracted from mouse aorta using RIPA buffer (cat. no. P0013B; Beyotime Institute of Biotechnology) and a BCA protein concentration kit (cat. no. P0010; Beyotime Institute of Biotechnology) was used for protein determination. The procedures used were conducted as described previously (28). The gelatinolytic activities of MMP-2 and MMP-9 were quantified via densitometric analysis by Azure Biosystems capture software v1.6 (29).

Statistical analysis. All the experiments were repeated three times. Data were analyzed using SPSS 19.0 software (IBM Corp.) and are presented as the mean \pm SEM. The significant differences in mean values among groups was examined via one-way ANOVA followed by Tukey's post hoc test. $\mathrm{P}<0.05$ was considered to indicate a statistically significant difference.

\section{Results}

ADAM10 reduces pathological injury to the abdominal aortic wall in a mouse AAA model. A total of 2 weeks after elastase perfusion, a severe AAA developed with significant dilation of the infrarenal aortic lumen in the AAA groups. Ultrasound examination revealed that the largest diameters of the aorta were significantly increased compared with those in the Sham groups, while they were significantly decreased in the ADAM10 groups (Fig. 1A and B).

H\&E staining identified that ADAM10 decreased the injury to the abdominal aortic wall. A normal abdominal aortic wall was shown in sham group (Fig. 1C-a and d). The significant injury in elastic laminae presented with flattening, fragmentation and degeneration in the medial layer, accompanied with the thickened and remodeled aortic adventitia in the AAA groups (Fig. 1C-b and e). ADAM10 significantly reduced elastin degradation in the aortic media, and the elastic lamellae partially recovered its wavy structures, thereby maintaining the integrity of the aortic intima in the ADAM10 groups (Fig. 1C-c and f). EVA staining revealed the normal form of elastin (Fig. 1D-a and d). Moreover, elastin in the aortic adventitia was thickened (Fig. 1D-b and e), which was reduced by ADAM10 (Fig. 1D-c and f).

ADAM10 reduces the inflammatory response of the abdominal aortic wall in a mouse AAA model. The inflammatory responses in the experimental model was examined via histopathologic analysis. Immunohistochemical staining demonstrated that there was only a few $\mathrm{CD}^{+} 8^{+}$inflammatory cells in the normal aortic wall (Fig. 2A-a and d), while the 
Table I. Primers for reverse transcription-quantitative PCR analysis.

\begin{tabular}{|c|c|}
\hline Gene & Sequences \\
\hline \multirow[t]{2}{*}{ RAGE } & Forward: 5'-GCTGTAGCTGGTGGTCAGAACA-3' \\
\hline & Reverse: 5'-CCCCTTACAGCTTAGCACAAGTG-3' \\
\hline \multirow[t]{2}{*}{ HMGB1 } & Forward: 5'-ATGGGCAAAGGAGATCCTA-3' \\
\hline & Reverse: 5'-ATTCATCATCATCATCTTCT-3' \\
\hline \multirow[t]{2}{*}{$\mathrm{NF}-\kappa \mathrm{B}$} & Forward: 5'-GCATTCTGACCTTGCCTATCT-3' \\
\hline & Reverse: 5'-CTCCAGTCTCCGAGTGAAGC-3' \\
\hline \multirow[t]{2}{*}{ MMP-2 } & Forward: 5'-CCCTGGTGGCTGGAGGCTCT-3' \\
\hline & Reverse: 5'-AACGGGGTCCCACGTCCCAA-3' \\
\hline \multirow[t]{2}{*}{ MMP-9 } & Forward: 5'-GCATCCGAGCAAGAAGACAAC-3' \\
\hline & Reverse: 5'-CCCGACACACAGTAAGCATTC-3' \\
\hline \multirow[t]{2}{*}{ IL-1 $\beta$} & Forward: 5'-TTGACGGACCCCAAAAGATG-3' \\
\hline & Reverse: 5'-AGAAGGTGCTCATGTCCTCA-3' \\
\hline \multirow[t]{2}{*}{ TNF- $\alpha$} & Forward: 5'-TCTCATCAGTTCTATGGCCC-3' \\
\hline & Reverse: 5'-GGGAGTAGACAAG GTACAAC-3' \\
\hline \multirow[t]{2}{*}{ MCP-1 } & Forward: 5'-TTAAAAACCTGGATCGGAACCAA-3' \\
\hline & Reverse: 5'-GCATTAGCTTCAGATTTACGGGT-3' \\
\hline \multirow[t]{2}{*}{$\beta$-actin } & Forward: 5'-GTGGGCCGCTCTAGGCACCAA-3' \\
\hline & Reverse: 5'-CTCTTTGATGTCACGCACGATTTC-3' \\
\hline
\end{tabular}

HMGB1, high-mobility-group box 1; RAGE, receptor for advanced glycation end products; MCP-1, monocyte chemoattractant protein-1 .

inflammatory cells accumulated and infiltrated into the aortic wall in the AAA groups (Fig. 2A-b and e), and this phenomenon was attenuated in the ADAM10 groups compared with the AAA groups (Fig. 2A-c and f). The increased number of $\mathrm{CD} 8^{+}$macrophages in the aortic wall in the AAA groups increased significantly compared with sham groups, which was significantly attenuated in the ADAM10 groups (Fig. 2B). Furthermore, ADAM10 decreased the expression levels of the proinflammatory mediators, including IL-1 $\beta$, TNF- $\alpha$ and monocyte chemoattractant protein-1, in the aortic wall of the AAA as shown via RT-qPCR analysis (Fig. 2C).

ADAM10 inhibits the expression of MMPs. RT-qPCR analysis demonstrated that the mRNA expression levels of MMP-2 and MMP-9 were significantly increased in the AAA groups, but were significantly downregulated in the ADAM10 groups on day 14 (Fig. 3A).

Gelatin zymography examination revealed that the activities of MMP-2 and MMP-9 were notably increased in the AAA groups and were significantly downregulated in the aneurysmal wall in the ADAM10 groups on day 14 (Fig. 3B).

ADAM10 reduces serum HMGB1 levels, while increasing serum sRAGE levels in a mouse AAA model. Serum HMGB1 and sRAGE levels changed in an opposite manner in the AAA group. In the AAA group, serum HMGB1 was significantly increased as detected via ELISA, and this effect was reduced in the ADAM10 group (Fig. 4A). Furthermore, a lower level of serum sRAGE was detected in the AAA group, while a significant increase in serum sRAGE levels was observed in the ADAM10 group (Fig. 4B).
ADAM10 decreases aortic wall HMGB1, RAGE and NF- $\kappa B$ expression in the AAA model. The mRNA and protein expression levels of HMGB1, RAGE and NF- $\kappa$ B were detected in the aneurysm walls via RT-qPCR and western blotting, respectively. The RT-qPCR results demonstrated that the mRNA expression level of HMGB1 was higher in the AAA group, which was significantly downregulated by ADAM10. Moreover, the mRNA expression level of RAGE was higher in the AAA group, which was significantly reduced by ADAM10. It was also found that the mRNA expression level of NF- $\kappa \mathrm{B}$ was higher in the AAA group, which was decreased significantly by ADAM10 (Fig. 5A). The western blotting results were consistent with those of the RT-qPCR. The protein expression levels of HMGB1 and RAGE, and the ratio of the phosphorylation of NF- $\kappa \mathrm{B}$ p $65 / \mathrm{NF}-\kappa \mathrm{B}$ p65 were higher in the AAA group, and were significantly decreased in the ADAM10 group (Fig. 5B).

ADAM10 inhibits the progression rate of small AAAs in a mouse model. Ultrasound examination showed the largest diameters of the aorta on day 0,3, 7 and 14 after infusion (Fig. 6A). In the ADAM10-treated group, after 4 days, the growth curve of the AAA began to alter, showing a downward trend, and the maximum aortic diameter was significantly decreased after 3 and 10 days of continuous treatment (Fig. 6B). This indicated that ADAM10 treatment was able to significantly limit the progression rate of small AAAs.

\section{Discussion}

In the present study, to test the hypothesis that the inflammatory mediator ADAM10 is an important factor in the 
A
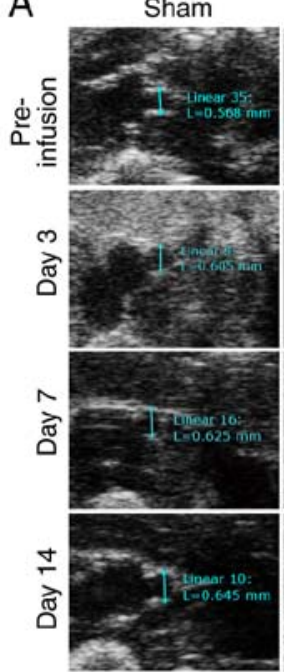

B
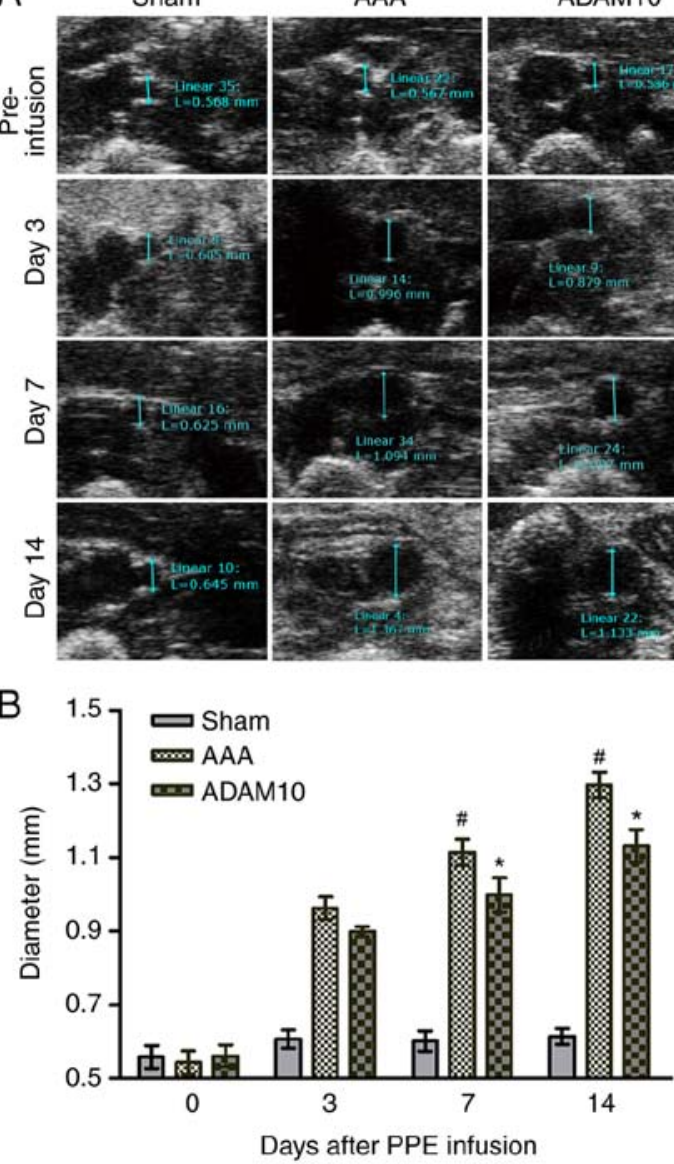

C

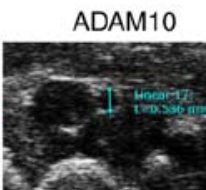

a

Sham
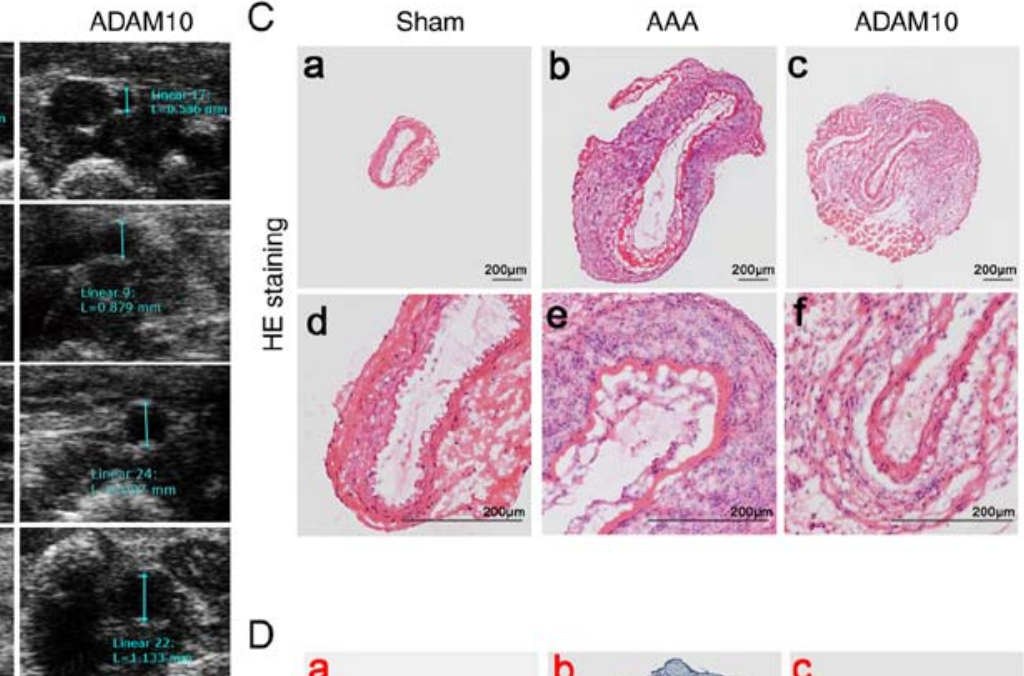

D

a
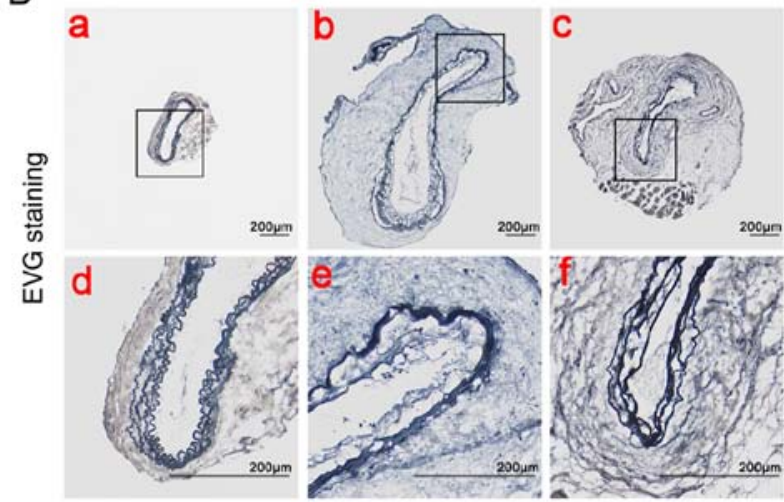

Figure 1. ADAM10 reduces pathological injury to the abdominal aortic wall in a mouse AAA model. (A) Representative ultrasonographic images of aortas from the different groups before infusion and on day 14 after infusion. The blue bar indicates the diameter of the aorta. (B) Quantitative analysis of the diameters of the aortas on days 0,3,7 and 14 after infusion in different groups. (Ca) A normal abdominal aortic wall was shown in sham group in low magnification. (Cb) The injury in elastic laminae and aortic adventitia was shown in the AAA groups in low magnification. (Cc) ADAM10 significantly reduced the injury in the aortic wall in the ADAM10 groups in low magnification. (Cd) A normal abdominal aortic wall was shown in sham groups in high magnification. (Ce) The injury in elastic laminae and aortic adventitia was shown in the AAA groups in high magnification. (Cf) ADAM10 significantly reduced the injury in the aortic wall in the ADAM10 groups in high magnification. (Da) EVA staining showed the normal form of elastin in sham groups in low magnification. (Db) EVA staining showed the thickened elastin in the aortic adventitia in AAA groups in low magnification. (Dc) EVA staining showed reduced elastin in ADAM10 groups in low magnification. (Dd) EVA staining showed the normal form of elastin in sham groups in high magnification. (De) EVA staining showed the thickened elastin in the aortic adventitia in AAA groups in high magnification. (Df) EVA staining showed reduced elastin in ADAM10 groups in high magnification. Scale bars, $200 \mu \mathrm{m}$. All the data are expressed as the mean \pm SEM; $n=7$ per group. ${ }^{\#} \mathrm{P}<0.05$ vs. sham group; ${ }^{*} \mathrm{P}<0.05$ vs. AAA group. Sham group, the mouse received intraaortic infusions of saline alone; AAA group, the mouse treatment with intraaortic elastase infusion; ADAM10 group, the mouse received ADAM10 treatment after intraaortic elastase infusion. EVG, elastica van Gieson; AAA, abdominal aortic aneurysm; ADAM10, ADAM metallopeptidase domain 10.

pathophysiology of AAA, a mouse AAA model was established by incubating the infrarenal aorta with pancreatic elastase. The results demonstrated that ADAM10 inhibited HMGB1/RAGE/NF- $\kappa \mathrm{B}$ signaling and MMP activity in the pathogenesis of pancreatic elastase-induced AAA.

AAA is described as a degenerative and dilated disease of the abdominal aorta and small, asymptomatic AAAs currently have no favorable therapeutic options, although it has been demonstrated in animal and clinical models that inflammatory processes have an important role in the pathophysiology of AAA (5). Macrophages accumulating and infiltrating the aneurysmal wall cause tissue injury, producing and responding to inflammatory mediators, and they release proteases, such as Cathepsin S protein and MMPs, resulting in ECM remodeling during the formation of AAAs (30). There are numerous drugs that have been tested in animals and clinical trials with the goal of inhibiting the development of AAAs, such as statins (31), $\beta$-adrenoceptor antagonists (32), renin-angiotensin system inhibitors (33) and doxycycline (34). However, at present, there is no established drug therapy that can efficiently inhibit the development of AAAs or reverse small AAAs (35).

The present study selected a mouse AAA model induced by pancreatic elastase perfusion, the pathological changes of which are considered to be the closest to the pathophysiological changes of human AAAs (36). The aneurysm wall has obvious inflammatory responses (37), which is consistent with the purpose of the current study. This experimental mouse model has been widely accepted and applied to study the pathophysiology of AAA (38). To examine its therapeutic effects, ADAM10 was intraperitoneally injected into the mice on the first day after the AAA was induced and the treatment continued for 10 days. The present results suggested that ADAM10 can significantly alleviate the development of AAAs, which was reflected by the observation that ADAM10 inhibited the dilatation of the infrarenal aorta. After ADAM10 
A

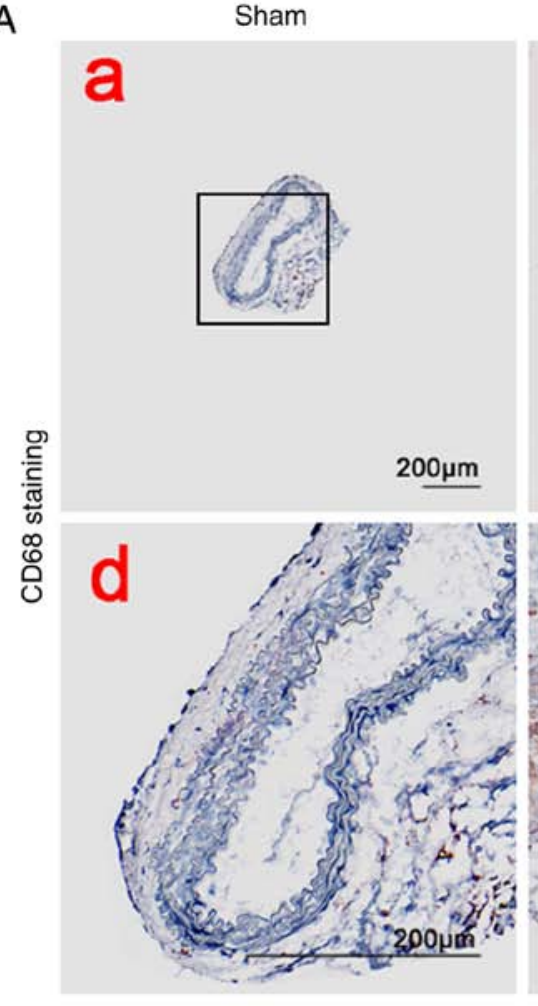

B

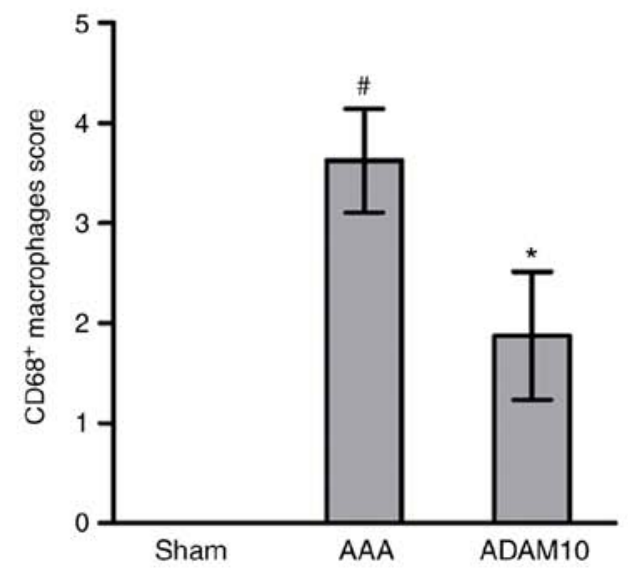

AAA
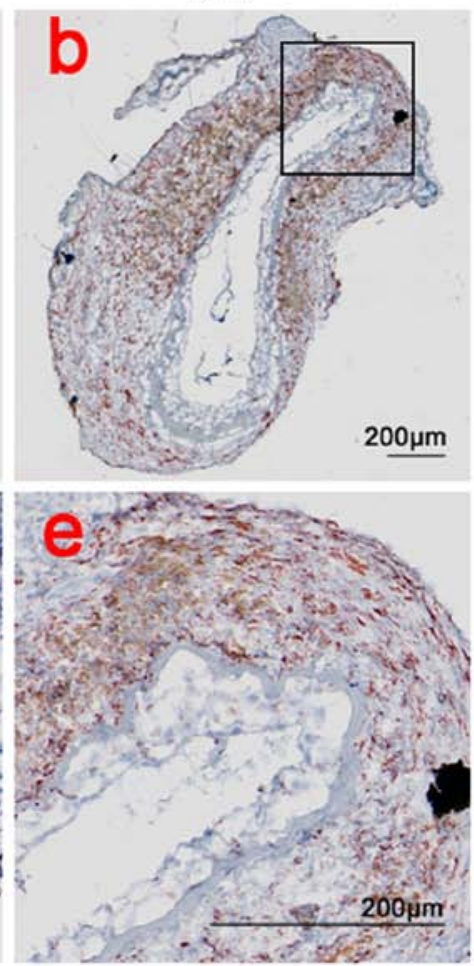

ADAM10
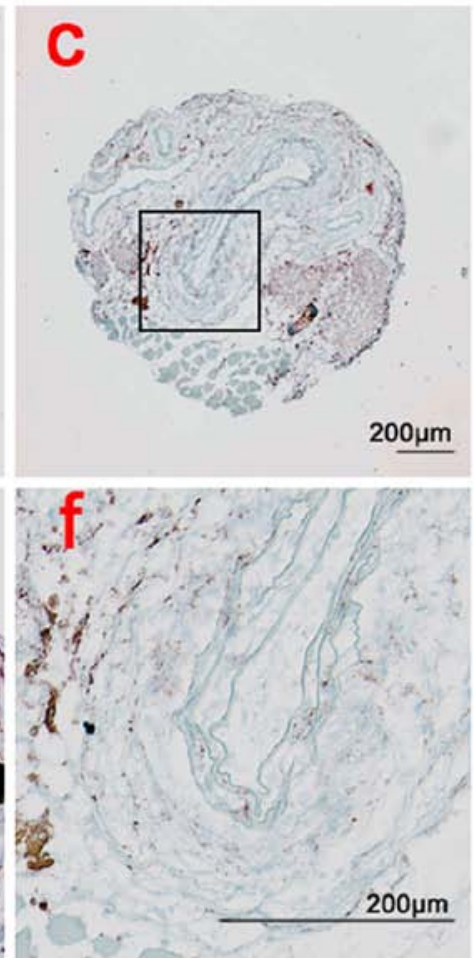

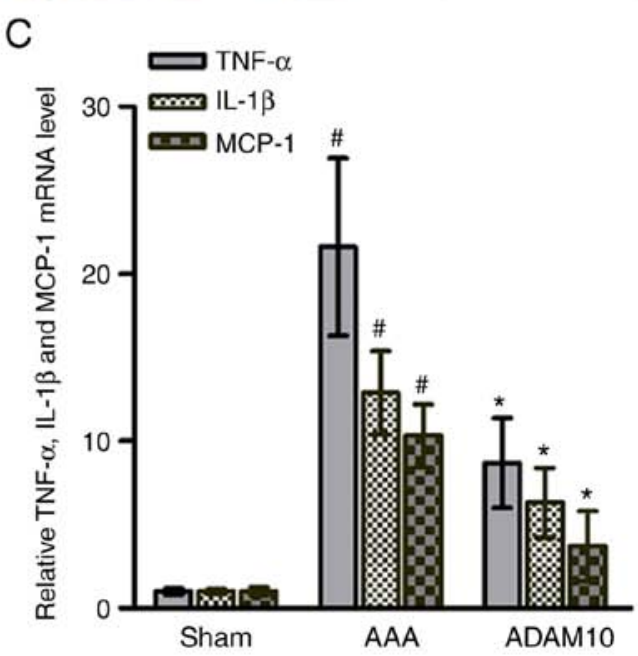

Figure 2. ADAM10 reduces the inflammatory response of the abdominal aortic wall in a mouse AAA model. (A) Representative micrographs of immunohistochemical staining of cluster of differentiation $\mathrm{CD}^{+} 8^{+}$macrophages in different groups. (Aa) CD68+ inflammatory cells in the normal aortic wall in sham groups in low magnification. (Ab) The CD68+ inflammatory cells accumulated and infiltrated into the aortic wall in the AAA groups in low magnification. (Ac) The infiltration of CD68+ inflammatory cells was attenuated in ADAM10 groups in low magnification. (Ad) CD68+ inflammatory cells in the normal aortic wall in sham groups in low magnification. (Ae) The CD68+ inflammatory cells accumulated and infiltrated into the aortic wall in the AAA groups in low magnification. (Af) The infiltration of CD68+ inflammatory cells was attenuated in ADAM10 groups in low magnification. Scale bars, $200 \mu \mathrm{m}$. (B) Quantitative analysis of the scores of CD68 ${ }^{+}$cells. (C) Reverse transcription-quantitative PCR analysis showing the expression levels of IL-1 $\beta$, TNF- $\alpha$ and MCP- 1 in the different groups. ${ }^{\#} \mathrm{P}<0.05$ vs. sham group; "P<0.05 vs. AAA group. The relative mRNA levels of TNF- $\alpha$, IL- $1 \beta$ and MCP- 1 were normalized to those of $\beta$-actin. MCP-1, monocyte chemoattractant protein-1; AAA, abdominal aortic aneurysm; ADAM10, ADAM metallopeptidase domain 10.

treatment, the flattening, fragmentation and degeneration of the aortic intimal elastic laminae and remodeling of the aortic adventitia were significantly relieved compared with those of the AAA group. All of these results suggested that ADAM10 had an obvious preventive effect on the progression of AAA, which provides a basis for its clinical transformation and its use for the inhibition of AAAs. The mechanism may be mediated via downregulation of HMGB/RAGE/NF- $\mathrm{B}$ signaling.

HMGB-1, a typical damage-associated molecular pattern protein released from various cells, such as macrophages, smooth muscle cells, endothelial cells, cardiomyocytes, epithelial cells and neurons (39), serves a pivotal role in multiple cardiovascular diseases, inflammatory and infectious disorders $(40,41)$. It performs its biological function by combining with receptors, including RAGE (42). In the present experiment, the expression of HMGB1 was increased in the circulation at both the transcriptional and translational levels in AAA model mice compared with the Sham group, and HMGB1 has been shown to activate and amplify inflammatory effector mechanisms (43). RAGE is the cellular target receptor for HMGB1, and the binding 
A
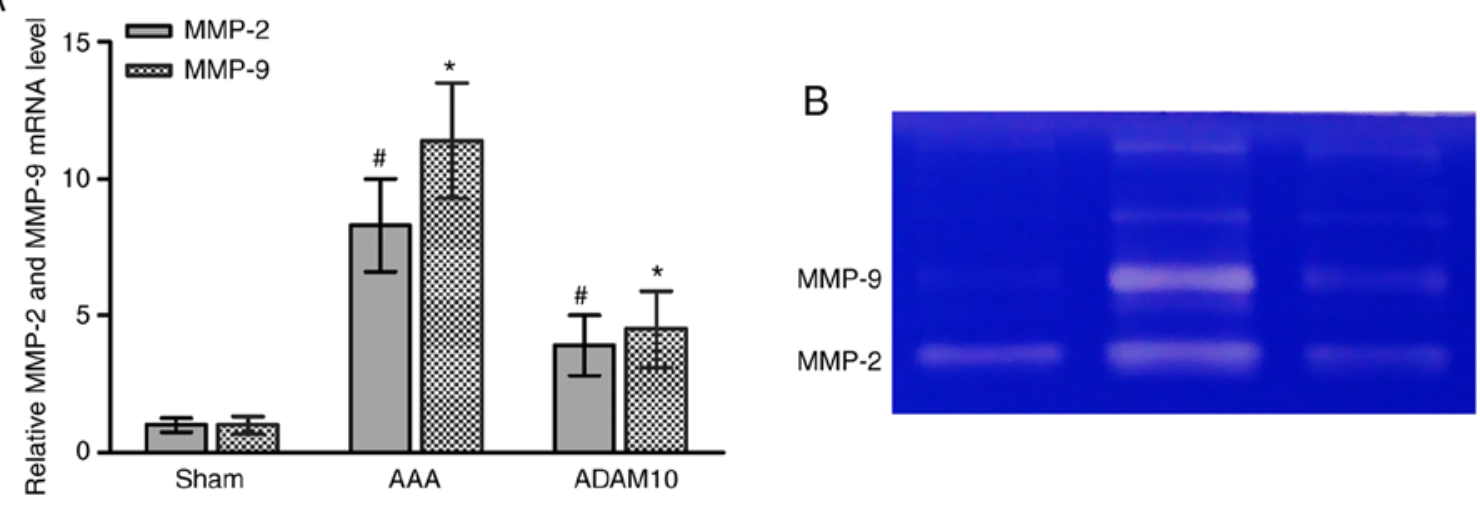

Figure 3. ADAM10 inhibits the expression of MMPs. (A) Reverse transcription-quantitative PCR analysis showing the mRNA expression levels of MMP-2 and MMP-9 on day 14. (B) Gelatin zymography examination showing the activities of MMP-2 and MMP-9 on day 14 . "P<0.05 vs. sham group; ${ }^{*} \mathrm{P}<0.05$ vs. AAA group. AAA, abdominal aortic aneurysm; ADAM10, ADAM metallopeptidase domain 10.

A

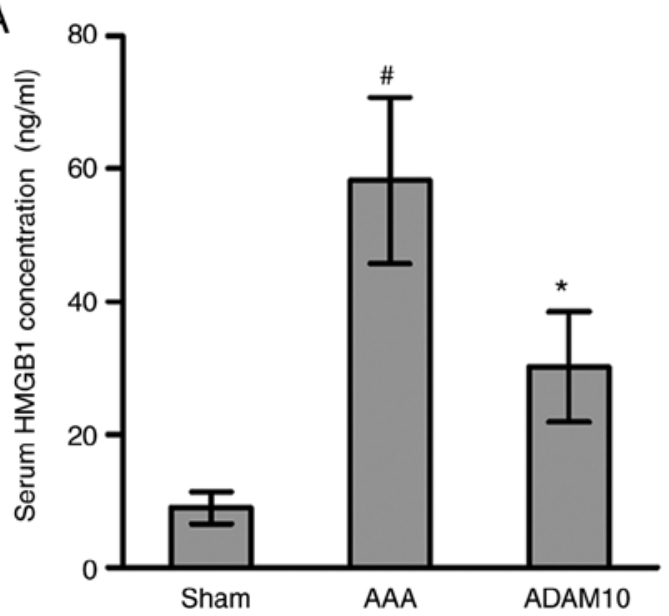

B

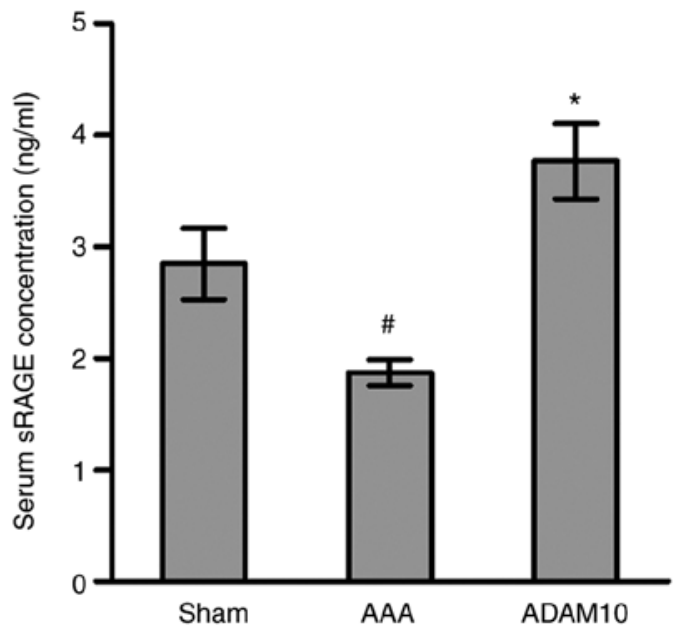

Figure 4. ADAM10 reduces serum HMGB1 levels, while increasing serum sRAGE levels in a mouse AAA model. ELISA showing the levels of serum (A) HMGB1 and (B) sRAGE. "P<0.05 vs. sham group; ${ }^{*} \mathrm{P}<0.05$ vs. AAA group. AAA, abdominal aortic aneurysm; ADAM10, ADAM metallopeptidase domain 10; sRAGE, soluble receptor for advanced glycosylation end products; HMGB1, high mobility group box 1 .

of HMGB1 to the RAGE pathway is associated with activating and modulating the inflammatory pathological process (44); therefore, the HMGB1/RAGE signaling axis may play a critical role in the development of AAAs.

RAGE has been shown to be involved in the pathological process of chronic inflammatory diseases, such as atherosclerosis, AD, inflammatory bowel disease, rheumatoid arthritis, amyloidosis and late diabetic vascular complications (45). In general, the extracellular domain is critical and is responsible for binding to the specific ligand, and the cytosolic tail domain is essential for transmitting RAGE signaling from the cell surface to the downstream targets involved in the progression of inflammatory responses (46). Ligand binding of fl-RAGE can activate and amplify inflammatory states (47) as sRAGE lacks the transmembrane and C-terminal cytoplasmic domain of fl-RAGE, and thus, is unable to mediate intracellular signaling, acting as a 'decoy' receptor. sRAGE competitively combines with the same ligand and prevents its binding to fl-RAGE, without conducting signals to the intracellular site to activate downstream signaling cascades $(22,48)$. Previous animal studies indicated that treatment of diabetic $\mathrm{RAGE}^{-/ /}$apoE ${ }^{-/}$mice suppressed inflammation of the blood vessels and oxidative stress, as well as attenuated atherosclerosis development (49). ADAM10-mediated ectodomain shedding of the extracellular domain of RAGE simultaneously generates sRAGE, and serves a role in inhibiting the RAGE-mediated signaling pathway and alleviating inflammation (50). In support of this, in the current study, RAGE expression was increased in the AAA model group and was decreased in the ADAM10 group. Increasing serum sRAGE suggests that RAGE participates in the pathological development of AAA. Additionally, ADAM10 inhibited RAGE activity via ectodomain cleavage of functional fl-RAGE to produce more inactive sRAGE, acting as a decoy, competitively binding ligands and inhibiting its deleterious effects. The present finding was also consistent with previous studies examining inflammatory, autoimmune and infectious diseases (51-53).

$\mathrm{NF}-\kappa \mathrm{B}$ is a family implicated in numerous diseases, ranging from cancer to inflammatory and immune disorders (54). Inflammation serves an important role in contributing to the development of AAAs. Importantly, $\mathrm{NF}-\kappa \mathrm{B}$ also regulates inflammation (55). HMGB1/RAGE signaling stimulates a positive feedback loop, induces multiple downstream signaling 
A

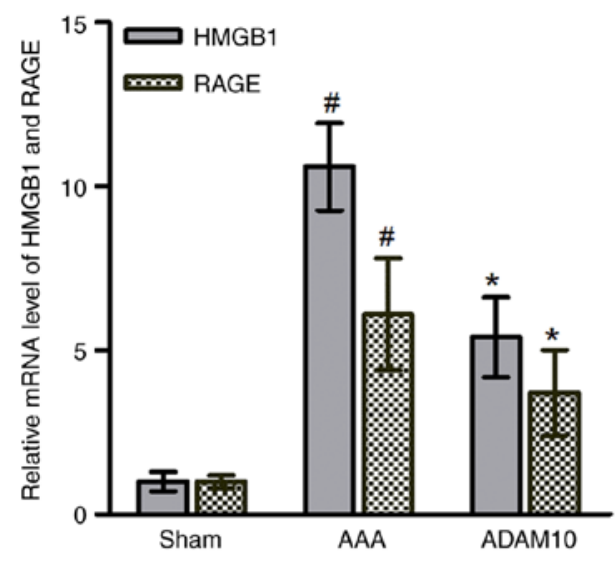

B
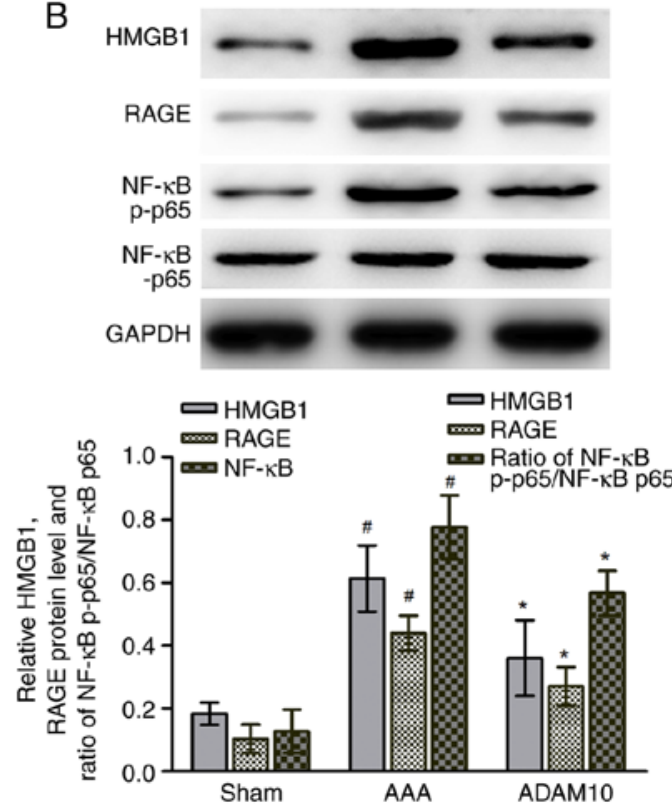

Figure 5. ADAM10 reduces aortic wall HMGB1, RAGE and NF- $\mathrm{kB}$ expression in the AAA model. (A) Reverse transcription-quantitative PCR analysis showing the mRNA expression levels of HMGB1 and RAGE on day 14. (B) Representative western blotting images and summarized data showing the protein

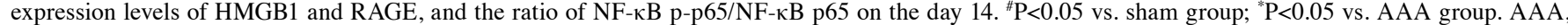
abdominal aortic aneurysm; ADAM10, ADAM metallopeptidase domain 10; RAGE, receptor for advanced glycosylation end products; HMGB1, high mobility group box 1; p-, phosphorylated.
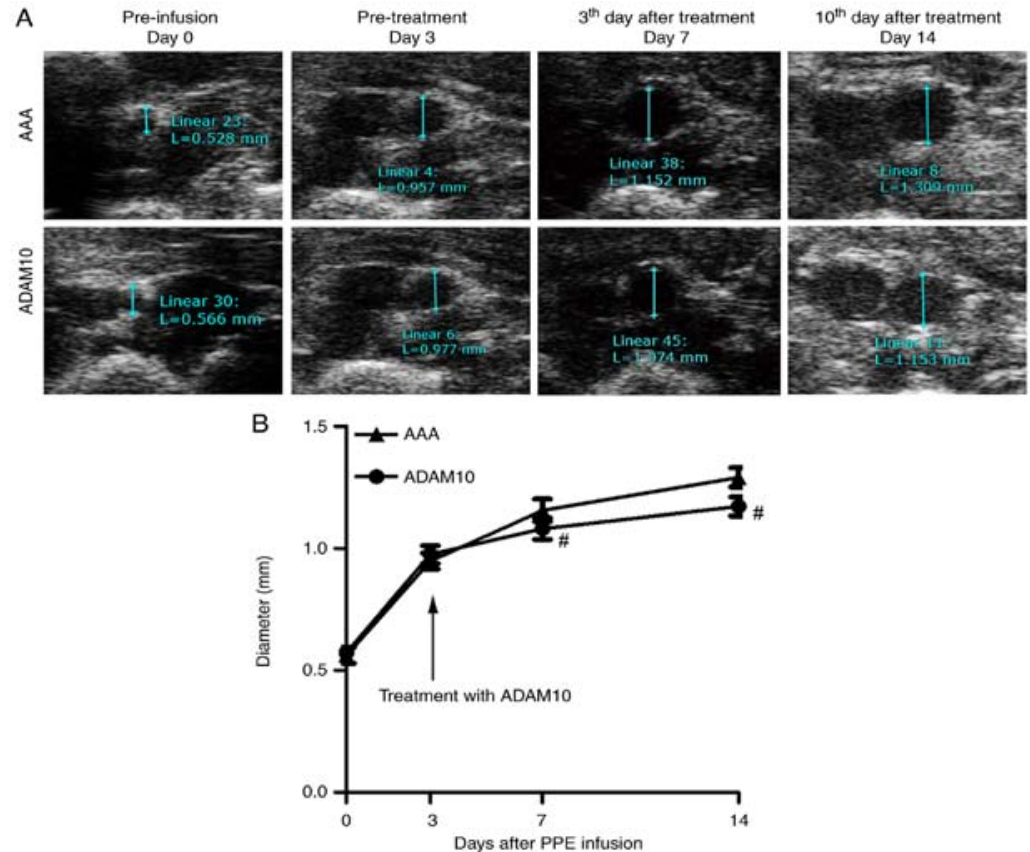

Figure 6. ADAM10 inhibits the progression rate of small AAAs in a mouse model. (A) Representative ultrasonographic images of aortas from the different groups on day $0,3,7$ and 14 after infusion. The blue bar indicates the diameter of the aorta. (B) Quantitative analysis of the diameter curve of the aortas on days $0,3,7$ and 14 after infusion in different groups. ${ }^{~} \mathrm{P}<0.05$ vs. AAA group. AAA, abdominal aortic aneurysm; ADAM10, ADAM metallopeptidase domain 10; PPE, porcine pancreatic elastase.

pathways, such as the stress-activated protein kinase/JNK, cell division cycle 42/Rac, p38 MAPK and ERK1/2, and activates the downstream proinflammatory transcription factor NF- $\mathrm{\kappa B}$, which subsequently induces the production of inflammatory factors $(55,56)$. Thus, the HMGB1/RAGE/NF- $\mathrm{KB}$ axis may be involved in the pathophysiology of AAA.
In the present study, typical AAA formation was observed in the AAA group, and an associated pathway (HMGB1/RAGE/NF- $\mathrm{kB}$ ) was activated, which was consistent with previous studies (55-59). The diameter of the AAA was significantly smaller and the HMGB1/RAGE/NF- $\kappa B$ pathway was inactivated in the ADAM10 group. Therefore, 
it was suggested that ADAM10 inhibited RAGE activity by cleaving fl-RAGE to non-functional sRAGE as a decoy receptor that neutralizes circulating ligands, thereby affecting HMGB1 binding with RAGE, and ultimately inhibiting the activity of the HMGB1/RAGE/NF- $\mathrm{BB}$ signaling axis and reducing inflammation in the AAA wall. ADAM10 decreased inflammatory factor induced aggregation by macrophages, T-cells and B-cells, and further reduced MMP-2 and MMP-9 production, thus alleviating the development of AAA. However, the results in the current study should be interpreted with caution since they may not completely reflect changes that are occurring on a functional level.

In conclusion, the present study demonstrated that ADAM10 alleviated the development of AAA in an intraluminal elastase-induced mouse AAA model and reversed small AAAs by inhibiting aortic inflammation via the HMGB1/RAGE/NF- $\mathrm{BB}$ pathway to further inhibit MMP activity. This study provides insight into the molecular mechanism and a potential therapeutic strategy for AAA.

\section{Acknowledgements}

Not applicable.

\section{Funding}

This research was funded by National Natural Science Foundation of China (grant nos. 81800409 and 81670435) and the Major Research and Development Program of Shandong (grant no. 2017GSF218074).

\section{Availability of data and materials}

The datasets used and/or analyzed during the current study are available from the corresponding author on reasonable request.

\section{Authors' contributions}

RQ, SC and GL were responsible for designing the study, collecting the data, performing the statistical analysis and drafting the manuscript. RQ, PG, KL, XF and HY collected the data, performed the statistical analysis and conducted the literature search. XW and GL supervised the project, helped to design the study, analyzed the data and wrote the manuscript. All authors confirm the authenticity of all the raw data. All authors have read and approved the final manuscript.

\section{Ethics approval and consent to participate}

This study was approved by the Animal Ethics Committee of Shandong University (approval no. 2018-015).

\section{Patient consent for publication}

Not applicable.

\section{Competing interests}

The authors declare that they have no competing interests.

\section{References}

1. Johnston KW, Rutherford RB, Tilson MD, Shah DM, Hollier L and Stanley JC: Suggested standards for reporting on arterial aneurysms. Subcommittee on Reporting Standards for Arterial Aneurysms, Ad Hoc Committee on Reporting Standards, Society for Vascular Surgery and North American Chapter, International Society for Cardiovascular Surgery. J Vasc Surg 13: 452-458, 1991.

2. Sampson UK, Norman PE, Fowkes FG, Aboyans V, Yanna Song, Harrell FE Jr, Forouzanfar MH, Naghavi M, Denenberg JO, McDermott MM, et al: Global and regional burden of aortic dissection and aneurysms: Mortality trends in 21 world regions, 1990 to 2010. Glob Heart 9: 171-180.e10, 2014.

3. Ghaferi AA, Birkmeyer JD and Dimick JB: Variation in hospital mortality associated with inpatient surgery. N Engl J Med 361: 1368-1375, 2009.

4. Shang T, Ran F, Qiao Q, Liu Z and Liu CJ: Tanshinone IIA attenuates elastase-induced AAA in rats via inhibition of MyD88-dependent TLR-4 signaling. Vasa 43: 39-46, 2014.

5. Golledge J: Abdominal aortic aneurysm: Update on pathogenesis and medical treatments. Nat Rev Cardiol 16: 225-242, 2019.

6. Raffort J, Lareyre F, Clément M, Hassen-Khodja R, Chinetti G and Mallat Z: Monocytes and macrophages in abdominal aortic aneurysm. Nat Rev Cardiol 14: 457-471, 2017.

7. Neeper M, Schmidt AM, Brett J, Yan SD, Wang F, Pan YC, Elliston K, Stern D and Shaw A: Cloning and expression of a cell surface receptor for advanced glycosylation end products of proteins. J Biol Chem 267: 14998-15004, 1992.

8. Kierdorf $\mathrm{K}$ and Fritz G: RAGE regulation and signaling in inflammation and beyond. J Leukoc Biol 94: 55-68, 2013.

9. Piperi C, Adamopoulos C, Dalagiorgou G, Diamanti-Kandarakis E and Papavassiliou AG: Crosstalk between advanced glycation and endoplasmic reticulum stress: Emerging therapeutic targeting for metabolic diseases. J Clin Endocrinol Metab 97: 2231-2242, 2012.

10. Huttunen HJ, Fages $\mathrm{C}$ and Rauvala $\mathrm{H}$ : Receptor for advanced glycation end products (RAGE)-mediated neurite outgrowth and activation of NF-kappaB require the cytoplasmic domain of the receptor but different downstream signaling pathways. J Biol Chem 274: 19919-19924, 1999.

11. Santilli F, Vazzana N, Bucciarelli LG and Davì G: Soluble forms of RAGE in human diseases: Clinical and therapeutical implications. Curr Med Chem 16: 940-952, 2009.

12. van Zoelen MA, Yang H, Florquin S, Meijers JC, Akira S, Arnold B, Nawroth PP, Bierhaus A, Tracey KJ and van der Poll T: Role of toll-like receptors 2 and 4 , and the receptor for advanced glycation end products in high-mobility group box 1-induced inflammation in vivo. Shock 31: 280-284, 2009.

13. Paudel YN, Angelopoulou E, Piperi C, Balasubramaniam VRMT, Othman I and Shaikh MF: Enlightening the role of high mobility group box 1 (HMGB1) in inflammation: Updates on receptor signalling. Eur J Pharmacol 858: 172487, 2019.

14. Andersson $\mathrm{U}$, Yang $\mathrm{H}$ and Harris $\mathrm{H}$ : High-mobility group box 1 protein (HMGB1) operates as an alarmin outside as well as inside cells. Semin Immunol 38: 40-48, 2018.

15. Oozawa S, Mori S, Kanke T, Takahashi H, Liu K, Tomono Y, Asanuma M, Miyazaki I, Nishibori M and Sano S: Effects of HMGB1 on ischemia-reperfusion injury in the rat heart. Circ J 72: 1178-1184, 2008.

16. Andrassy M, Volz HC, Igwe JC, Funke B, Eichberger SN, Kaya Z, Buss S, Autschbach F, Pleger ST, Lukic IK, et al: High-mobility group box-1 in ischemia-reperfusion injury of the heart. Circulation 117: 3216-3226, 2008.

17. Hamada T, Torikai M, Kuwazuru A, Tanaka M, Horai N, Fukuda T, Yamada S, Nagayama S, Hashiguchi K, Sunahara N, et al: Extracellular high mobility group box chromosomal protein 1 is a coupling factor for hypoxia and inflammation in arthritis. Arthritis Rheum 58: 2675-2685, 2008.

18. Golledge J, Muller J, Daugherty A and Norman P: Abdominal aortic aneurysm: Pathogenesis and implications for management. Arterioscler Thromb Vasc Biol 26: 2605-2613, 2006.

19. Saftig P and Reiss K: The 'A Disintegrin And Metalloproteases' ADAM10 and ADAM17: Novel drug targets with therapeutic potential? Eur J Cell Biol 90: 527-535, 2011.

20. Donners MM, Wolfs IM, Olieslagers S, Mohammadi-Motahhari Z, Tchaikovski V, Heeneman S, van Buul JD, Caolo V, Molin DG, Post MJ and Waltenberger J: A disintegrin and metalloprotease 10 is a novel mediator of vascular endothelial growth factor-induced endothelial cell function in angiogenesis and is associated with atherosclerosis. Arterioscler Thromb Vasc Biol 30: 2188-2195, 2010. 
21. Maretzky T, Reiss K, Ludwig A, Buchholz J, Scholz F, Proksch E, de Strooper B, Hartmann D and Saftig P: ADAM10 mediates E-cadherin shedding and regulates epithelial cell-cell adhesion, migration, and beta-catenin translocation. Proc Natl Acad Sci USA 102: 9182-9187, 2005

22. Raucci A, Cugusi S, Antonelli A, Barabino SM, Monti L, Bierhaus A, Reiss K, Saftig P and Bianchi ME: A soluble form of the receptor for advanced glycation endproducts (RAGE) is produced by proteolytic cleavage of the membrane-bound form by the sheddase a disintegrin and metalloprotease 10 (ADAM10). FASEB J 22: 3716-3727, 2008.

23. Jiao T, Yao Y, Zhang B, Hao DC, Sun QF, Li JB, Yuan C, Jing B, Wang YP and Wang HY: Role of MicroRNA-103a targeting ADAM10 in abdominal aortic aneurysm. Biomed Res Int 2017: 9645874, 2017.

24. National Research Council (US) Institute for Laboratory Animal Research: Guide for the care and use of laboratory animals. Washington (DC), National Academies Press, 1996.

25. Anidjar S, Salzmann JL, Gentric D, Lagneau P, Camilleri JP and Michel JB: Elastase-induced experimental aneurysms in rats. Circulation 82: 973-981, 1990.

26. Zhang F, Kent KC, Yamanouchi D, Zhang Y, Kato K, Tsai S, Nowygrod R, Schmidt AM and Liu B: Anti-receptor for advanced glycation end products therapies as novel treatment for abdominal aortic aneurysm. Ann Surg 250: 416-423, 2009.

27. Livak KJ and Schmittgen TD: Analysis of relative gene expression data using real-time quantitative PCR and the 2(-Delta Delta C(T)) method. Methods 25: 402-408, 2001.

28. Li G, Yang L, Yuan H, Liu Y, He Y, Wu X and Jin X: Cold-inducible RNA-binding protein plays a central role in the pathogenesis of abdominal aortic aneurysm in a murine experimental model. Surgery 159: 1654-1667, 2016

29. Snoek-van Beurden PA and Von den Hoff JW: Zymographic techniques for the analysis of matrix metalloproteinases and their inhibitors. Biotechniques 38: 73-83, 2005.

30. Murray PJ and Wynn TA: Protective and pathogenic functions of macrophage subsets. Nat Rev Immunol 11: 723-737, 2011.

31. Shiraya S, Miyake T, Aoki M, Yoshikazu F, Ohgi S, Nishimura M, Ogihara T and Morishita R: Inhibition of development of experimental aortic abdominal aneurysm in rat model by atorvastatin through inhibition of macrophage migration. Atherosclerosis 202: $34-40,2009$.

32. Lindholt JS, Henneberg EW, Juul S and Fasting H: Impaired results of a randomised double blinded clinical trial of propranolol versus placebo on the expansion rate of small abdominal aortic aneurysms. Int Angiol 18: 52-57, 1999.

33. Morris DR, Cunningham MA, Ahimastos AA, Kingwell BA, Pappas E, Bourke M, Reid CM, Stijnen T, Dalman RL, Aalami OO, et al: TElmisartan in the management of abDominal aortic aneurYsm (TEDY): The study protocol for a randomized controlled trial. Trials 16: 274, 2015

34. Meijer CA, Stijnen T, Wasser MN, Hamming JF, van Bockel JH and Lindeman JH; Pharmaceutical Aneurysm Stabilisation Trial Study Group: Doxycycline for stabilization of abdominal aortic aneurysms: A randomized trial. Ann Intern Med 159: 815-823, 2013.

35. Kokje VB, Hamming JF and Lindeman JH: Editor's Choice-pharmaceutical management of small abdominal aortic aneurysms: A systematic review of the clinical evidence. Eur J Vasc Endovasc Surg 50: 702-713, 2015.

36. Busch A, Holm A, Wagner N, Ergün S, Rosenfeld M, Otto C, Baur J, Kellersmann R and Lorenz U: Extra- and intraluminal elastase induce morphologically distinct abdominal aortic aneurysms in mice and thus represent specific subtypes of human disease. J Vasc Res 53 49-57, 2016.

37. Busch A, Chernogubova E, Jin H, Meurer F, Eckstein HH, Kim M and Maegdefessel L: Four surgical modifications to the classic elastase perfusion aneurysm model enable haemodynamic alterations and extended elastase perfusion. Eur J Vasc Endovasc Surg 56 102-109, 2018

38. Johnston WF, Salmon M, Su G, Lu G, Ailawadi G and Upchurch GR Jr: Aromatase is required for female abdominal aortic aneurysm protection. J Vasc Surg 61: 1565-74.e1-e4, 2015.

39. Wu H, Li R, Pei LG, Wei ZH, Kang LN, Wang L, Xie J and Xu B: Emerging role of high mobility group Box-1 in thrombosis-related diseases. Cell Physiol Biochem 47: 1319-1337, 2018.

40. Bae JS: Role of high mobility group box 1 in inflammatory disease: Focus on sepsis. Arch Pharm Res 35: 1511-1523, 2012.

41. Ding HS and Yang J: High mobility group box-1 and cardiovascular diseases. Saudi Med J 31: 486-489, 2010.
42. van Beijnum JR, Buurman WA and Griffioen AW: Convergence and amplification of toll-like receptor (TLR) and receptor for advanced glycation end products (RAGE) signaling pathways via high mobility group B1 (HMGB1). Angiogenesis 11: 91-99, 2008.

43. Hreggvidsdottir HS, Ostberg T, Wähämaa H, Schierbeck H, Aveberger AC, Klevenvall L, Palmblad K, Ottosson L, Andersson U and Harris HE: The alarmin HMGB1 acts in synergy with endogenous and exogenous danger signals to promote inflammation. J Leukoc Biol 86: 655-662, 2009.

44. Sims GP, Rowe DC, Rietdijk ST, Herbst R and Coyle AJ: HMGB1 and RAGE in inflammation and cancer. Annu Rev Immunol 28 367-388, 2010

45. Bierhaus A, Humpert PM, Morcos M, Wendt T, Chavakis T, Arnold B, Stern DM and Nawroth PP: Understanding RAGE, the receptor for advanced glycation end products. J Mol Med (Berl) 83: 876-886, 2005

46. Dattilo BM, Fritz G, Leclerc E, Kooi CW, Heizmann CW and Chazin WJ: The extracellular region of the receptor for advanced glycation end products is composed of two independent structural units. Biochemistry 46: 6957-6970, 2007.

47. Basta G, Lazzerini G, Massaro M, Simoncini T, Tanganelli P, Fu C, Kislinger T, Stern DM, Schmidt AM and De Caterina R Advanced glycation end products activate endothelium through signal-transduction receptor RAGE: A mechanism for amplification of inflammatory responses. Circulation 105: 816-822, 2002.

48. Sung JY, Chung W, Kim AJ, Kim HS, Ro H, Chang JH, Lee HH and Jung JY: Calcitriol treatment increases serum levels of the soluble receptor of advanced glycation end products in hemodialysis patients with secondary hyperparathyroidism. Tohoku J Exp Med 230: 59-66, 2013.

49. Soro-Paavonen A, Watson AM, Li J, Paavonen K, Koitka A, Calkin AC, Barit D, Coughlan MT, Drew BG, Lancaster GI, et al: Receptor for advanced glycation end products (RAGE) deficiency attenuates the development of atherosclerosis in diabetes. Diabetes 57: 2461-2469, 2008

50. Zhang L, Bukulin M, Kojro E, Roth A, Metz VV, Fahrenholz F, Nawroth PP, Bierhaus A and Postina R: Receptor for advanced glycation end products is subjected to protein ectodomain shedding by metalloproteinases. J Biol Chem 283: 35507-35516, 2008.

51. Detzen L, Cheng B, Chen CY, Papapanou PN and Lalla E: Soluble forms of the receptor for advanced glycation endproducts (RAGE) in periodontitis. Sci Rep 9: 8170, 2019.

52. Ramasamy R and Schmidt AM: Schmidt, Soluble RAGE: Therapy and biomarker in unraveling the RAGE axis in chronic disease and aging. Biochem Pharmacol 79: 1379-1386, 2010

53. Lee EJ and Park JH: Receptor for advanced glycation endproducts (RAGE), its ligands, and soluble RAGE: Potential biomarkers for diagnosis and therapeutic targets for human renal diseases. Genomics Inform 11: 224-229, 2013

54. Baud V and Karin M: Is NF-kappaB a good target for cancer therapy? Hopes and pitfalls. Nat Rev Drug Discov 8: 33-40, 2009.

55. Nakashima H, Aoki M, Miyake T, Kawasaki T, Iwai M, Jo N, Oishi M, Kataoka K, Ohgi S, Ogihara T, et al: Inhibition of experimental abdominal aortic aneurysm in the rat by use of decoy oligodeoxynucleotides suppressing activity of nuclear factor kappaB and ets transcription factors. Circulation 109: 132-138, 2004

56. Chen YJ, Chan DC, Chiang CK, Wang CC, Yang TH, Lan KC, Chao SC, Tsai KS, Yang RS, Liu SH, et al: Advanced glycation end-products induced VEGF production and inflammatory responses in human synoviocytes via RAGE-NF- $\kappa$ B pathway activation. J Orthop Res 34: 791-800, 2016.

57. Medeiros MC, Frasnelli SC, Bastos Ade S, Orrico SR and Rossa C Jr: Modulation of cell proliferation, survival and gene expression by RAGE and TLR signaling in cells of the innate and adaptive immune response: Role of p38 MAPK and NF-KB. J Appl Oral Sci 22: 185-193, 2014

58. Lappas M, Permezel M and Rice GE: Advanced glycation endproducts mediate pro-inflammatory actions in human gestational tissues via nuclear factor-kappaB and extracellular signal-regulated kinase 1/2. J Endocrinol 193: 269-277, 2007.

59. Hofmann MA, Drury S, Fu C, Qu W, Taguchi A, Lu Y, Avila C, Kambham N, Bierhaus A, Nawroth P, et al: RAGE mediates a novel proinflammatory axis: A central cell surface receptor for S100/calgranulin polypeptides. Cell 97: 889-901, 1999.

This work is licensed under a Creative Commons Attribution-NonCommercial-NoDerivatives 4.0 International (CC BY-NC-ND 4.0) License. 